\title{
RECONFIGURATION OF DISCRETE EVENT SYSTEM CONTROLLERS WITH DYNAMIC SENSING SET
}

\author{
Jing Liu and Houshang Darabi
}

\author{
University of Illinois at Chicago, Department of Mechanical and Industrial Engineering
}

\begin{abstract}
This paper presents a finite automaton model to describe the control reconfiguration of discrete event systems (DES) with respect to the dynamic changes of observation means, especially when the changes are not acceptable for the conventional observability theory. The model includes other classes of events besides the regular DES events, such as repair and failure events of the observation means. Given a regular DES controller, through a systematic procedure it is extended to include the effect of those events on the control. The potential application of this work is the optimization of the reconfiguration strategies. Copyright (C) 2005 IFAC
\end{abstract}

Keywords: supervisory control, observability, sensor failures, formal methods.

\section{INTRODUCTION}

This paper models the control reconfiguration of DES with respect to the change of observation means. Specifically, the effect of the reconfiguration strategies on DES when the change of observation means is not acceptable for the conventional observability theory is addressed.

The investigation on the observability theory of DES was triggered by two parallel and original works of (Lin and Wonham, 1988) and (Cieslak, et al., 1988). The DES observability is critical for the DES controller as the controller takes the control actions according to what it observes from the DES.

It is assumed that for each event, there is a sensor reporting its occurrence to the controller. If a sensor is not working properly or its communication channel with the controller fails, the controller cannot obtain the information regarding the occurrence of the corresponding event. In this case, from the controller's point of view, this event is unobservable. Once the sensor and/or communication channel are repaired, that event becomes observable again.

In practice, the sensors or communication channels can be broken down or repaired at any time during the control. In a sense, in certain state the controller may "see" a different set of events than it does in the initial state. In other words, the observability of events changes over time. In conventional observability theory of the DES (Lin and Wonham, 1988), it is assumed that the set of observable events remains unchanged. Furthermore, there are some events which are critical for keeping the right control - the "must-be-observed" events. However, if these events are not seen by the controller, the controlled DES will go out of control. But there are some occasions where the controller can survive even if some of the "must-be-observed" events are not observed according to (Darabi, et al., 2003). Here they propose a control switching policy to discover an appropriate control policy and switch to the new policy on the fly. The search for the new control policy and the switching actions are performed by another control agent called the mega-controller. The proposed switching theory in this work is based on the set of observable projections originally introduced in (Haji-Valizadeh and Loparo 1996). Therefore if a new control policy is found when the set of observable events changes, then this policy can survive for infinite time given that the observable events set does not change again after the switch. Such policies, called infinite time policies, have some limitations according to (Liu and Darabi 2004). In fact the infinite time policies cannot provide the controller maximum survival time subject to the dynamic observable event set.

In (Liu and Darabi 2004) the authors have developed the class of finite time policies. This class is a superset of the infinite time policies introduced by (Darabi, et al., 2003). This class, in addition to the infinite time policies, consists of all control policies that are feasible for a finite duration of time. A finite time observation policy is good for the current state, but may not be good for other states. Using finite time policies provides a more general solution for the 
controller reconfiguration upon the change of event observability. In addition, (Liu and Darabi 2004) offers a new reconfiguration strategy, so called control feedback adjustment, to resolve control conflicts.

One of the problems generated by the work (Liu and Darabi 2004) is the implementation of the control reconfiguration strategies through a mega-controller. The mega-controller interacts with the DES controller to adapt the control to the dynamic changes of observation means. In this paper, the authors develop a finite automaton model to describe combined behavior of the mega-controller and the DES controller. By this model, the reader can have a full picture of how the mega-controller evolves upon the change of observation means. This finite automaton includes other classes of events besides the regular DES events. For example repair and failure events of sensors can change the state of this finite automaton. Our development starts from the regular DES controlled finite automaton and through a systematic procedure it extends the regular automaton to include the behavior of additional events. One of the main applications of the extended finite automaton (not discussed in this paper) is in optimizing the reconfiguration decisions made by the mega-controller.

The paper is organized as follows. Section 2 gives the preliminaries. Section 3 provides the algorithm to generate the extended finite automaton. Section 4 presents an illustrative example for building the extended model, and section 5 concludes the paper and discusses the future research.

\section{PRELIMINARIES}

According (Ramadge and Wonham 1987), a DES is modeled by finite automaton $G=\left(Q, \Sigma, \delta, q_{0}, Q_{m}\right)$, where $Q$ is the set of states, $\Sigma$ is the finite set of events (which can be partitioned into two disjoint subsets, controllable events set $\Sigma_{c}, \quad$ and uncontrollable events set $\Sigma_{u c}$ ), $\delta: Q \times \Sigma \rightarrow Q$ is the transition functions, $q_{0}$ is the initial state, and $Q_{m} \subseteq Q$ is the set of marked states. $G$ is said to be blocking if $\overline{L_{m}} \subset L$, and nonblocking if $\overline{L_{m}}=L$, where $L=\left\{s \in \Sigma^{*}: \delta\left(q_{0}, s\right)\right.$ is defined $\}$ is the language generated by $G$ ( $\Sigma^{*}$ is the set of finite length strings of $\Sigma)$. $G$ models the uncontrolled plant. The supervisor $\mathbf{S}$ (or controller) interacts with $G$ (or plant) in a closed loop manner (Fig. 1), and it assures that the plant does not violate a given set of specifications. The specifications are the conditions that the designers wish to impose on the plant. Mathematically, $\quad \mathbf{S}=(S, \Phi) \quad, \quad$ where

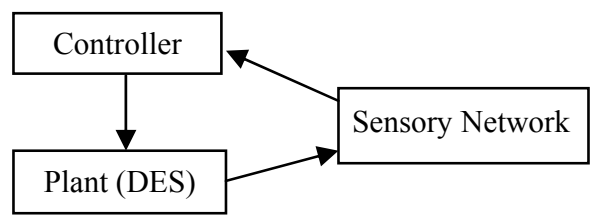

Fig. 1. DES control loop

$S=\left(X, \Sigma, f, x_{0}, X_{m}\right)$ is a deterministic automaton with state set $X$, initial state $x_{0}$, a marked subset, $X_{m} \subseteq X$ and transition function $f: X \times \Sigma \rightarrow X$; $\Phi: X \times \Sigma \rightarrow\{1,0, d c\}$ is the feedback function. $\Phi(x, \sigma)=1(0)$ indicates that the control action at state $x$ is to enable (disable) event $\sigma$. "dc" is an abbreviation for "don't care", which implies that the enabling or disabling of $\sigma$ at $x$ doesn't affect the behavior of $G . \Gamma: X \rightarrow 2^{\Sigma}$ is the active event function, and is defined as $\Gamma(x)=\{\sigma: f(x, \sigma)$ is defined $\}$ for all $x \in X$. In other words, $\Gamma(x)$ includes all the events that are enabled by $\mathbf{S}$ at state $x$. The controlled plant language, so called coupled language, is shown by $K=L(\mathbf{S} / G)$.

\section{EXTENDED CONTROLLER FINITE AUTOMATON MODEL}

It is assumed that a DES and its controller, as stated above, are given. It is also assume that there is a sensor associated with each event reporting its occurrence to the controller. Therefore, the sensor status change results in change in event observability. To develop the algorithm that generates the finite automata model with extended sensory events and reconfiguration decisions, some definitions are first provided.

The sensor status of an event $\sigma, s s_{\sigma}$, can be failed, represented by 0 and working, represented by 1 . The change of sensor status is triggered by two sets of events, sensor repair event set, $R$ and sensor breakdown event set $B . b_{\sigma} \in B, r_{\sigma} \in R$ are the sensor breakdown and repair event for $\sigma$ respectively.

An extended state $y$ is represented by " $\bar{X}_{-} S S$ " where $\bar{X} \subset X$ and $S S=\left(s s_{\sigma_{1}}, s s_{\sigma_{2}}, \cdots, s s_{\sigma_{i}}, \cdots, s s_{\sigma_{n}}\right)$ is the sensor status vector ( $s s_{\sigma_{i}}$ is the sensor status of event $\sigma_{i}$ in $\left.\Sigma=\left\{\sigma_{1}, \sigma_{2}, \cdots, \sigma_{i}, \cdots, \sigma_{n}\right\}\right)$. As defined before the element of $S S$ are 0 's or 1 's.

Extended state $y:=\bar{X}_{-} S S$ is a conflict state if there exist $x_{1}, x_{2} \in \bar{X}$ and $\sigma \in \Sigma$ such that $\Phi\left(x_{1}, e\right)=1$, $\Phi\left(x_{2}, e\right)=0$. 


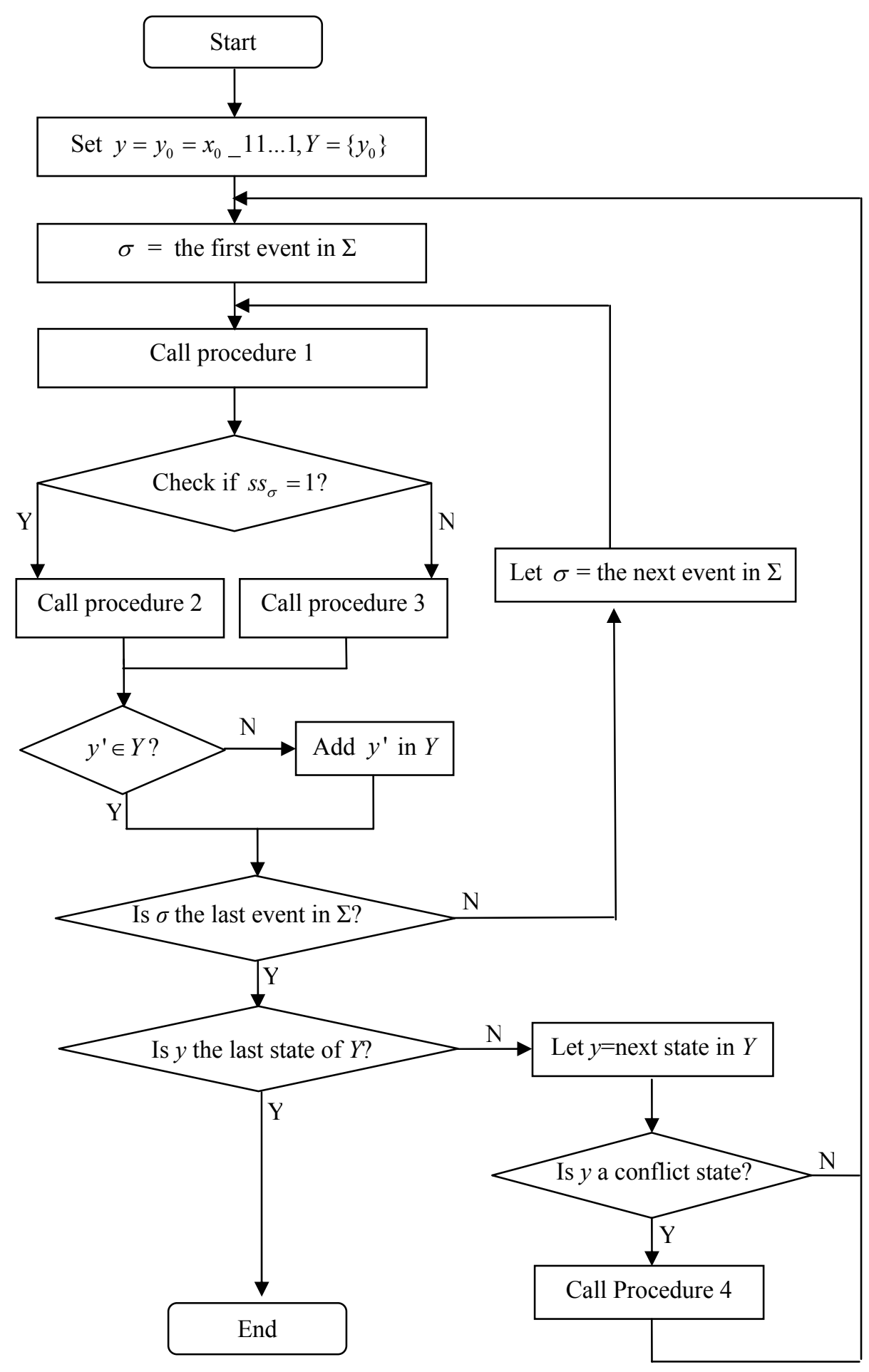

Fig. 2. Flow chart for building reconfigured controller $\Theta$

Accordingly, define the conflict event set of the conflict state by $C E(y)=\left\{\sigma \mid \exists x_{1}, x_{2} \in \bar{X}, \sigma \in \Sigma\right.$, $\left.\Phi\left(x_{1}, \sigma\right)=1, \Phi\left(x_{2}, \sigma\right)=0\right\}$, the enabling state set for the conflict event $\sigma \in C E(y)$ by $E S(y)=\{x \mid x \in \bar{X}, \sigma \in C E(y), \Phi(x, \sigma)=1\}$, and the disabling state set for the conflict event $\sigma \in C E(y)$ by $D S(y)=\{x \mid x \in \bar{X}, \sigma \in C E(y), \Phi(x, \sigma)=0\}$.

In order to have a full representation of the controller reconfiguration evolution, consider all the possible sensor status changes at any state. Given a controller $\mathbf{S}=(S, \Phi), \quad$ and $S$ is five-tuple automaton $S=\left(X, \Sigma, f, x_{0}, X_{m}\right)$, the extended controller automaton with a six-tuple automaton $\Theta=\left(Y, E, g, y_{0}, Y_{m}\right.$, inf $)$ is modeled. The elements in $\Theta$ are illustrated as follows:

(1) $Y$ - the set of extended states

The members of $Y$ are the states of the extended automaton and are generated using the flowchart in Figure 2. 
(2) $E$ - event space

$$
E=\Sigma \bigcup B \bigcup R
$$

(3) $y_{0}-$ initial state

$y_{0}:=x_{0} S S$

(4) $Y_{m}$ - marked subset

$Y_{m}=\left\{y \mid y:=x_{-} S S, x \in X_{m}\right\}$.

(5) inf - infeasible state

The infeasible state is any state that is reached from state $y_{0}$ through string $s$ such that $s \in L$ but $s \notin K$.

(6) $g-$ transition function

$g: Y \times E \rightarrow Y \bigcup\{$ inf $\}$

Accordingly, define active event function as $A E: Y \rightarrow 2^{E}$ and $A E(y)=\{e: g(y, e)$ is defined $\}$.

Figure 2 shows the algorithm for constructing $\Theta$. The inputs to these algorithms include the regular controller $\mathbf{S}$ and the initial extended state $y_{0}$. All the procedures used in Figure 2 are based on the occurrence of events in $\Sigma$ and the sensory events.

Procedure 1: called for every event $\sigma \in \Sigma$, let transition function $g(y, \sigma)=y^{\prime}$ and $y^{\prime}:=\bar{X}_{-}^{\prime} S S^{\prime}$ is computed as follows,

a) $\bar{X}_{1}=\{f(x, \sigma) \mid x \in \bar{X}, f(x, \sigma)$ is defined. $\}$;

b) $\bar{X}_{2}=\left\{f(x, t) \mid x \in \bar{X}_{1}, t=\left\{\sigma \mid \sigma \in \Sigma \text { and } s s_{\sigma}=0\right\}^{*}\right.$, $f(x, t)$ is defined. $\}$;

c) $\bar{X}^{\prime}=\bar{X}_{1} \cup \bar{X}_{2}, S S^{\prime}=S S, y^{\prime}:=\left(\bar{X}_{1} \cup \bar{X}_{2}\right)_{-} S S$.

d) Add $\sigma$ to $A E(y)$.

Procedure 2: called when $s s_{\sigma}=1$.

(1) Add $b_{\sigma}$ to $A E(y)$

(2) $g\left(y, b_{\sigma}\right)=y^{\prime}$, the algorithm for computing $y^{\prime}$ is as follows,

a) $\bar{X}^{\prime}=\{f(x, t) \mid x \in \bar{X}$, $\left.t=\left\{\sigma^{\prime} \mid \sigma^{\prime} \in \Sigma, s s_{\sigma^{\prime}}=0 \text { or } \sigma^{\prime}=\sigma\right\}^{*}\right\}$

b) $y^{\prime}:=\bar{X}_{-}^{\prime} S S^{\prime}$ where the element $s s_{\sigma^{\prime}}=\left\{\begin{array}{cc}0, & \text { if } \sigma^{\prime}=\sigma ; \\ s s_{\sigma}, & \text { otherwise. }\end{array}\right.$

Procedure 3: called when $s s_{\sigma}=0$.

(1) Add $r_{\sigma}$ to $A E(y)$.

(2) $g\left(y, b_{\sigma}\right)=y^{\prime}, y^{\prime}:=\bar{X} \_S S^{\prime}$ where the element $s s_{\sigma^{\prime}}=\left\{\begin{array}{cc}1, & \text { if } \sigma^{\prime}=\sigma ; \\ s s_{\sigma}, & \text { otherwise. }\end{array}\right.$

Procedure 4 (Feedback Adjustment): called when in a conflict state. At the conflict state $y:=\bar{X}_{-} S S$, there are two ways to adjust the feedback to resolve the conflict:

(1) Enable the conflict event $\sigma \in C E(y)$ at all the states in $D S(y) . g(y, \sigma)=y^{\prime}$, and $g(y, \sigma)=\inf$ where $y^{\prime}:=\bar{X}_{-}^{\prime} S S^{\prime}$ and $\bar{X}^{\prime}=\{f(x, \sigma) \mid x \in E S(y)\}$. This makes the reconfigured controller automaton $\Theta$ nondeterministic. At state $x \in D S(y)$, enabling $\sigma$ causes the controller to enter an infeasible state.

(2) Disable the conflict event $\sigma \in C E(y)$ at all the states in $D S(y)$. Remove even $\sigma$ from $A E(y)$. If $|A E(y)|=0$, then state $y$ is a deadlock.

Discussion The four procedures deal with the event occurrences and conflict resolving. Events include the regular events $(\Sigma)$, sensor breakdown events $(B)$ and sensor repair events $(R)$. At an extended state $y:=\bar{X}_{-} S S$, consider all the events that possibly can happen. First, every event in $\Sigma$ is examined to find the ones that can take place. Then, it is investigated if the sensor breakdown or repair events are possible to happen or not according to the current sensor status. For doing so, three rules are used. (1) Event $\sigma \in \Sigma$ can happen if and only if there is a state $x \in \bar{X}$ where $\sigma$ is active. (2) Sensor breakdown event $b_{\sigma}$ can happen if and only if $s s_{\sigma}=1$, i.e. sensor for event $\sigma$ can fail only when it is at work. (It is assumed that all sensors are working at the initial state). (3) Sensor repair event $r_{\sigma}$ can happen if and only if $s s_{\sigma}=0$, i.e. sensor for event $\sigma$ can be repaired only when it is failed. During this process, control conflict could come up as a result that some states requiring different feedback policy on certain events are aggregated together due to the unavailability of sensors. The procedures are explained one by one in the followings.

Procedure 1 handles the occurrence of events in $\Sigma$ (the event set of given controller). For the state set part $\bar{X}$, the states where that event is enabled are aggregated up, and the resultant state set are further combined with the states that cannot be differentiated due to the sensor failure. The sensor status vector remains the same.

Procedure 2 copes with the occurrence of sensor breakdown events. Once the failure takes place, the sensor cannot report the occurrence of corresponding event to the controller. The states that are reachable from the given state by the unobservable strings are aggregated together. The element in sensor status vector for that event changes from 1 to 0 accordingly.

Procedure 3 takes care of the case that sensor is failed. At this case, that sensor repair event is possible to happen. When it happens, it only affects the sensor status vector. 
Procedure 4 deals with the control conflict resolving. There are two ways to tackle the conflict, either enabling or disabling the events that cause the conflict. Which way is overweight the other one is different from case to case.

From the generation of $\Theta$ it is evident that the feedback adjustment at conflict states could generate states not reachable or not feasible. Taking different feedback policy adjustments affects the evolution path of the finite automaton $\Theta$, and thus affects the chance of leading to infeasible or blocking states. As mentioned in the introduction, the selection between different actions outlined by $\Theta$ requires an optimization model that uses $\Theta$ as its input. Another input to this model can be a reconfiguration criterion such as maximizing the probability of reaching to a marked state. However, the objective of this paper is not to address such an optimization framework.

\section{An Illustrative Example}

The controller automaton is shown in Figure 3, and the feedback of each state is listed in table 1. Event set $\Sigma=\left\{e_{1}, e_{2}, e_{3}\right\}$, and all are controllable. $c_{m}=-5$, $c_{I}=4$ and $c_{b}=2$.

For this example, configure the controller under the policy that event $e_{1}, e_{2}$ are to be enabled and $e_{3}$ is to be disabled if there is a conflict.

Due to the space limitation, the figure of the finite automaton of the reconfigured controller cannot be shown completely. Here only a part of the figure in Figure 4 is shown.

The evolution of the extended controller $\Theta$ is illustrated as follows,

(1) At the initial state 1_111,

- event $e_{1}$ is active, call procedure 1 .

Because $f\left(1, e_{3}\right)=3, g\left(1 \_111, e_{1}\right)=3 \_111$.

- $s s_{e_{2}}=1$, call procedure 2. Add $b_{e_{2}}$ to $A E\left(1_{-} 111\right)$.

Because $f\left(1, e_{2}\right)=2, g\left(1_{-} 111, b_{e_{1}}\right)=12_{-} 101$.

(2) At state 12_101,

State 12_101 is conflict, and $C E\left(12 \_101\right)=\left\{e_{1}\right\}$.

If $e_{1}$ is enabled at $D S\left(12_{-} 101\right)=\{2\}$,

then $g\left(12 \_101, e_{1}\right)=3{ }_{-} 101$, and $g\left(12 \_101, e_{1}\right)=i n f$.

(3) At state 3_111, $e_{3}$ is active, follow procedure $1, f\left(3, e_{3}\right)=4$, $g\left(3_{-} 111, e_{3}\right)=4 \_111$ which is the marked state.

(4) At state 34_110,

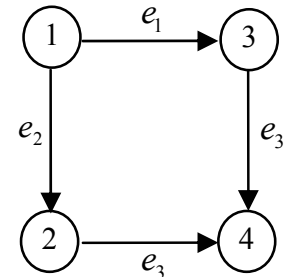

Fig. 3. Controller automaton of the illustrative example.

Table 1 Feedback table of the illustrative

\begin{tabular}{cccc}
\hline & $e_{1}$ & $e_{2}$ & $e_{3}$ \\
\hline 1 & 1 & 1 & $\mathrm{dc}$ \\
\hline 2 & 0 & $\mathrm{dc}$ & 1 \\
\hline 3 & $\mathrm{dc}$ & 0 & 1 \\
\hline 4 & $\mathrm{dc}$ & $\mathrm{dc}$ & 0 \\
\hline
\end{tabular}

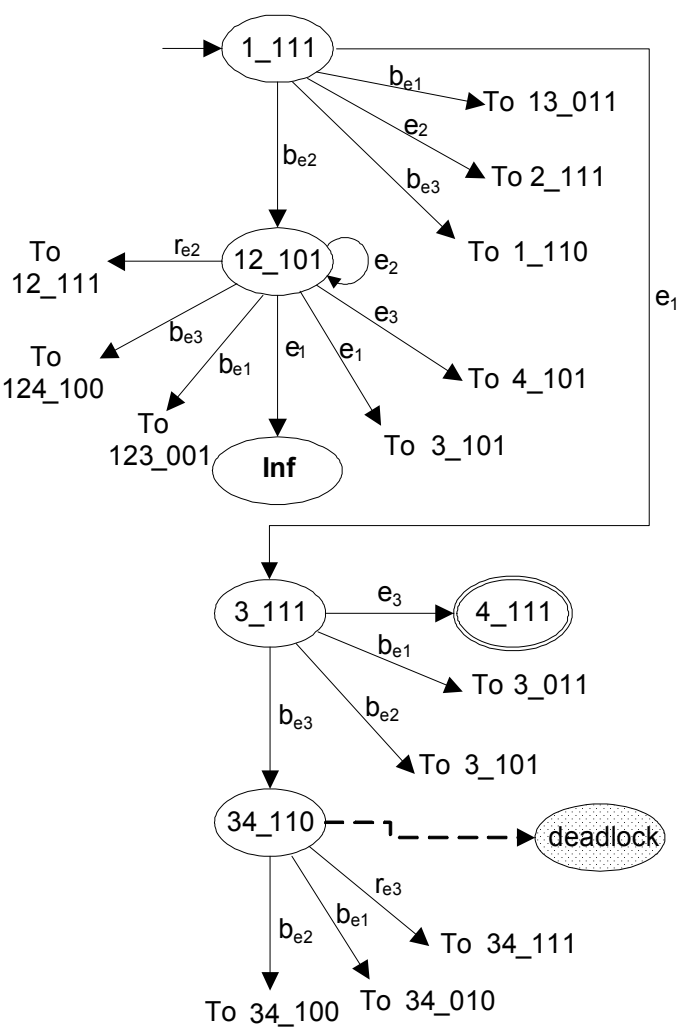

Fig. 4. Partial representation of the extended automaton $\Theta$

State 34_110 is conflict, and $C E\left(34 \_110\right)=\left\{e_{3}\right\}$. If $e_{3}$ is disabled at $E S\left(34 \_110\right)=\{3\}$, then $g\left(34 \_110, e_{3}\right)=\emptyset$, so there is a chance that state 34_110 becomes a deadlock itself.

The transitions between states are listed in Table 2 .

\section{CONCLUSION AND FUTURE RESEARCH}

This paper provides a formal model that embeds the controller reconfiguration options upon the change in 
Table 2. Transitions between states for illustrative example.

\begin{tabular}{|c|c|c|c|c|c|c|c|c|c|c|}
\hline \multirow{2}{*}{$\begin{array}{l}\text { State } \\
\text { Name }\end{array}$} & \multirow{2}{*}{$\begin{array}{c}\text { State } \\
\text { No. }\end{array}$} & \multicolumn{9}{|c|}{ Events } \\
\hline & & $e_{1}$ & $e_{2}$ & $e_{3}$ & $b_{e 1}$ & $b_{e 2}$ & $b_{e 3}$ & $r_{e 1}$ & $r_{\mathrm{e} 2}$ & $r_{\mathrm{e} 3}$ \\
\hline 1_111 & 1 & 3 & 2 & & 7 & 8 & 9 & & & \\
\hline 2_111 & 2 & & & 4 & 10 & 11 & 12 & & & \\
\hline 3_111 & 3 & & & 4 & 13 & 14 & 15 & & & \\
\hline 4_111 & 4 & & & & & & & & & \\
\hline inf & 5 & & & & & & & & & \\
\hline block & 6 & & & & & & & & & \\
\hline 13_011 & 7 & 7 & 5,10 & 16 & & 20 & 21 & 19 & & \\
\hline 12_101 & 8 & 5,14 & 8 & 17 & 20 & & 23 & & 22 & \\
\hline 1_110 & 9 & 15 & 12 & & 21 & 23 & & & & \\
\hline 2_011 & 10 & & & 16 & & 24 & 25 & 2 & & \\
\hline 2_101 & 11 & & & 17 & 24 & & 26 & & 2 & \\
\hline 24_110 & 12 & & & 6 & 25 & 26 & & & & 27 \\
\hline 3_011 & 13 & & & 16 & & 28 & 29 & 3 & & \\
\hline 3_101 & 14 & & & 17 & 28 & & 30 & & 3 & \\
\hline 34_110 & 15 & & & 6 & 29 & 30 & & & & 31 \\
\hline 4_011 & 16 & & & & & 32 & 33 & 4 & & \\
\hline 4_101 & 17 & & & & 32 & & 34 & & 4 & \\
\hline 4_110 & 18 & & & & 33 & 34 & & & & 4 \\
\hline 13_111 & 19 & 3 & 2,5 & 4 & 7 & 35 & 36 & & & \\
\hline 123_001 & 20 & 5,20 & 5,20 & 32 & & & 38 & 35 & 37 & \\
\hline 134_010 & 21 & 21 & 5,25 & 6 & & 38 & & 36 & & 39 \\
\hline 12_111 & 22 & 5,3 & 2 & 4 & 37 & 8 & 40 & & & \\
\hline 124_100 & 23 & 5,30 & 23 & 6 & 38 & & & & 40 & 41 \\
\hline 2_001 & 24 & & & 32 & & & 42 & 11 & 10 & \\
\hline 24_010 & 25 & & & 6 & & 42 & & 12 & & 43 \\
\hline 24_100 & 26 & & & 6 & 42 & & & & 12 & 44 \\
\hline 24_111 & 27 & & & 6 & 43 & 44 & 12 & & & \\
\hline 3_001 & 28 & & & 32 & & & 45 & 14 & 13 & \\
\hline 34_010 & 29 & & & 6 & & 45 & & 15 & & 46 \\
\hline 34_100 & 30 & & & 6 & 45 & & & & 15 & 47 \\
\hline 34_111 & 31 & & & 6 & 46 & 47 & 15 & & & \\
\hline 4_001 & 32 & & & & & & 48 & 17 & 16 & \\
\hline 4_010 & 33 & & & & & 48 & & 18 & & 16 \\
\hline 4_100 & 34 & & & & 48 & & & & 18 & 17 \\
\hline 123_101 & 35 & 5,14 & 5,35 & 17 & 20 & & 50 & & 49 & \\
\hline 134_110 & 36 & 15 & 5,12 & 6 & 21 & 50 & & & & 51 \\
\hline 123_011 & 37 & 5,37 & 5,10 & 16 & & 20 & 52 & 49 & & \\
\hline 1234_000 & 38 & 5,38 & 5,38 & 6 & & & & 50 & 52 & 53 \\
\hline 134_011 & 39 & 39 & 5,10 & 6 & & 53 & 21 & 51 & & \\
\hline 124_110 & 40 & 5,15 & 12 & 6 & 52 & 23 & & & & 54 \\
\hline 124_101 & 41 & 5,14 & 41 & 6 & 53 & & 23 & & 54 & \\
\hline 24_000 & 42 & & & 6 & & & & 26 & 25 & 55 \\
\hline 24_011 & 43 & & & 6 & & 55 & 25 & 27 & & \\
\hline 24_101 & 44 & & & 6 & 55 & & 26 & & 27 & \\
\hline 34_000 & 45 & & & 6 & & & & 30 & 29 & 56 \\
\hline 34_011 & 46 & & & 6 & & 56 & 29 & 31 & & \\
\hline 34_101 & 47 & & & 6 & 56 & & 30 & & 31 & \\
\hline 4_000 & 48 & & & & & & & 34 & 33 & 32 \\
\hline 123_111 & 49 & 5,3 & 2,5 & 4 & 37 & 35 & 57 & & & \\
\hline 1234_100 & 50 & 5,30 & 5,50 & 6 & 38 & & & & 57 & 58 \\
\hline 134_111 & 51 & 3 & 2,5 & 6 & 39 & 58 & 36 & & & \\
\hline
\end{tabular}

\begin{tabular}{c||c||c|c|c|c|c|c|c|c|c}
\hline \multirow{2}{*}{$\begin{array}{c}\text { State } \\
\text { Name }\end{array}$} & \multicolumn{1}{c||}{ State } & \multicolumn{10}{c}{ No. } & $e_{1}$ & $e_{2}$ & $e_{3}$ & $b_{e 1}$ & $b_{\mathrm{e} 2}$ & $b_{\mathrm{e} 3}$ & $r_{\mathrm{e} 1}$ & $r_{\mathrm{e} 2}$ & $r_{\mathrm{e} 3}$ \\
\hline 1234_010 & 52 & 5,52 & 5,25 & 6 & & 38 & & 57 & & 59 \\
\hline 1234_001 & 53 & 5,53 & 5,53 & 6 & & & 38 & 58 & 59 & \\
\hline $124 \_111$ & 54 & 3,5 & 2 & 6 & 59 & 41 & 40 & & & \\
\hline $24 \_001$ & 55 & & & 6 & & & 42 & 44 & 43 & \\
\hline 34_001 & 56 & & & 6 & & & 45 & 47 & 46 & \\
\hline 1234_110 & 57 & 5,15 & 5,12 & 6 & 52 & 50 & & & & 60 \\
\hline 1234_101 & 58 & 5,14 & 5,58 & 6 & 53 & & 50 & & 60 & \\
\hline 1234_011 & 59 & 5,59 & 5,10 & 6 & & 53 & 52 & 60 & & \\
\hline 1234_111 & 60 & 5,3 & 2,5 & 6 & 59 & 58 & 57 & & & \\
\hline
\end{tabular}

the availability of observation means. In this model, all the possible changes are considered. During the creation of the model, four control reconfiguration procedures are adopted which cover all the possible situations of sensor status changes. This model is useful for the studying the reaction of controller to the changes in the sensor status information. It can be also used for the optimization of reconfiguration strategies. The optimization problem cannot be solved directly by this model, but it could be converted to a Markov decision process that provides the optimal reconfiguration strategies. The discussion of the optimization framework will be the subject of our future research.

\section{REFERENCES}

Cieslak, R., C. Desclaux, A. S. Fawaz and P. Varaiya (1988). Supervisory control of discreteevent processes with partial observations. IEEE Transactions on Automatic Control, vol. 33, no. 3, pp. 249-260.

Darabi, H., M. A. Jafari and A. L. Buczak (2003). A control switching theory for supervisory control of discrete event systems. IEEE Transactions on Robotics and Automation, vol. 19, no.1, pp. 131137.

Haji-Valizadeh, A. K. and A. Loparo (1996). Minimizing the cardinality of an events set for supervisors of discrete-event dynamical systems. IEEE Transactions on Automatic Control, vol. 41, no. 11, pp. 1579-1593.

Lin, F. and W. A. Wonham (1988). On observability of Discrete event systems. Information Sciences, vol. 44, no. 3, pp. 173-198.

Liu, J. and H. Darabi (2004). Control Reconfiguration of Discrete Event Systems Controllers with Partial Observation. IEEE Transactions on Systems, Man and Cybernetics, Part B, vol. 34, no. 6, pp. 2262-2272.

Ramadge, P. J. and W. M. Wonham (1987). Supervisory control of a class of discrete event Processes. SIAM Journal of Control and Optimization, vol. 25, no.1, pp. 206-230. 